\title{
Targeting the cell cycle and the PI3K pathway: A possible universal strategy to reactivate innate tumor suppressor programmes in cancer cells
}

\author{
THÉRÈSE DAVID-PFEUTY ${ }^{1,3}$, MICHEL LEGRAVEREND ${ }^{2}$, ODILE LUDWIG ${ }^{2}$ and DAVID S. GRIERSON ${ }^{2}$ \\ ${ }^{1}$ UMR 146 and ${ }^{2}$ UMR 176 du CNRS, Institut Curie-Recherche, Bâtiment 110, \\ Centre Universitaire, 91405 Orsay Cedex, France
}

Received October 15, 2007; Accepted December 27, 2007

DOI: 10.3892/ijo_00000565

\begin{abstract}
Corruption of the $R b$ and $p 53$ pathways occurs in virtually all human cancers. This could be because it lends oncogene-bearing cells a surfeit of Cdk activity and growth, enabling them to elaborate strategies to evade tumorsuppressive mechanisms and divide inappropriately. Targeting both Cdk activities and the PI3K pathway might be therefore a potentially universal means to palliate their deficiency in cancer cells. We showed that the killing efficacy of roscovitine and 16 other purines and potentiation of roscovitine-induced apoptosis by the PI3K inhibitor, LY294002, decreased with increasing corruption of the $R b$ and $p 53$ pathways. Further, we showed that purines differing by a single substitution, which exerted little lethal effect on distant cell types in rich medium, could display widelydiffering cytotoxicity profiles toward the same cell types in poor medium. Thus, closely-related compounds targeting similar Cdks may interact with different targets that could compete for their interaction with therapeutically-relevant Cdk targets. In the perspective of clinical development in association with the $P I 3 K$ pathway inhibitors, it might thus be advisable to select tumor cell type-specific Cdk inhibitors on the basis of their toxicity in cell-culture-based assays performed at a limiting serum concentration sufficient to
\end{abstract}

Correspondence to: Dr Thérèse David-Pfeuty, ${ }^{3}$ Present address: UMR 8080, Bâtiment 440, Centre Universitaire, 91405 Orsay Cedex, France

E-mail: therese.pfeuty@u-psud.fr

Abbreviations: Rb, retinoblastoma; Ink4a, inhibitor of Cdk4; p53*, mutated p53; Arf, alternative reading frame; RTK, receptor tyrosine kinase; Cdk, cyclin-dependent kinase; PI3K, phosphatidyl inositol 3 kinase; PTEN, phosphatase and tensin homologue; mTOR, mammalian target of rapamycin; GSK-3, glycogen synthase kinase 3; SV40 LT, large T antigen of simian virus 40; HPV, human papillomavirus; IGF, insulin-like growth factor; rDNA, ribosomal DNA; Pol I/III, RNA polymerase I/III; RP, ribosomal protein

Key words: Rb, p53, cell cycle, cyclin-dependent kinase, cell growth, phosphatidyl inositol 3 kinase, anticancer strategy suppress their interaction with undesirable crossreacting targets whose range and concentration would depend on the cell genotype.

\section{Introduction}

Cancers arise from mutations that enable somatic cells to break tissue homeostasis and proliferate in settings where they would normally be sentenced to either exit the cell cycle or die (1). The retinoblastoma $(\mathrm{Rb})$ and p53 proteins appear as key components in homeostatic pathways (2). Rb is a powerful repressor of cell cycle-regulating gene transcription, whose sequential phosphorylation and inactivation by cyclin D- and cyclin E-Cdks is required for cells to depart from quiescence (3). p53 is a transcription factor that induces cell cycle exit or apoptosis upon activation by many stresses, including oncogenic insults $(2,4)$. Human cancers invariably harbor a functional alteration of one, or more, component(s) of the $R b$ and $p 53$ pathways and typically overexpress cyclins $\mathrm{D}$ or $\mathrm{E}$ and/or certain Cdks (2).

Targeting the cell cycle has thus emerged as a promising avenue for anticancer therapy, prompting the search for potent and selective Cdk inhibitors intended to stop tumor growth (5-7). Several ATP competitive Cdk inhibitors have now reached the stage of clinical trials (flavopiridol, UCN-01, CYC-202 and BMS-387032). Intriguingly, these compounds not only arrest cells in G1 and G2 but also trigger apoptosis in certain contexts. Such an unforeseen lethal effect could result from the fortuitous interaction of the drugs with a Cdkunrelated target. A more tantalizing hypothesis, however, is that $\mathrm{Cdk}$ inhibition is able to reactivate proapoptotic pathways silenced, and/or extinguish prosurvival pathways triggered by hyperactivated Cdks. Indeed, inhibition of Cdk 7 and Cdk9, that are specifically involved in transcriptional regulation, leads to the degradation of unstable mRNAs, including transcripts for apoptosis regulators (6). Also, Cdk2 inhibition is able to interfere with orderly Sphase progression, thereby promoting apoptosis via both p53-dependent and -independent mechanisms (8). A further key argument derives from the observation that roscovitine (9) induces both a nuclear p53 accumulation and p53independent nucleolar disassembly in human cultured cells (10). The roscovitine effects were fully reversible upon short- 
term treatment, transiently braking cell cycle progression, but became irreversible in longer term, ending in apoptosis, especially in tumor cells $(10,11)$. This suggests that roscovitine-sensitive Cdks not only control cell division, but also facilitate cell growth and mitigate p53 function in cell cycle arrest and apoptosis. They would further explain why oncogene-bearing cells that overexpress Cdks evade the $p 53$ pathway. Most importantly, they point to an alternative means by which hyperactivated Cdks might help tumor cells break tissue homeostasis. This involves a boosting of their translational capacity, providing tumor cells with a surfeit of growth and survival factors enabling them to ultimately divide inappropriately.

In addition to this, $\mathrm{Rb}$ and p53 also play a role in ribosome biogenesis (12), hinting that their inactivation could enforce growth via Cdk-dependent and -independent mechanisms. An important inference from this realization is that targeting both the protein synthetic machinery and the activity of Cdks may be a powerful, and perhaps universal, strategy to compensate for corruption of the $R b$ and $p 53$ pathways in cancer cells. Impediment of cell growth could be achieved by inhibition of the $P I 3 K$ pathway, which emerged recently as a central regulator of cell growth (13). Serum privation could also be used in in vitro assays as a stratagem to, in part, emulate effects of antiangiogenic compounds that deprive cells of their provision of growth and survival factors (14).

Our study revealed that both inhibition of protein kinases mTOR and PI3K (two central PI3K pathway components; 13) and serum starvation potentiates the lethal effects of roscovitine. The cytotoxicity enhancement, however, varied depending on both the mode of cotreatment and the tumor cell type. The investigation was extended to a series of purine-based compounds differing from each other and from roscovitine by the nature of the C- 2 and C- 6 substituents (15-17). We showed that purines differing by a single substitution, which exerted little lethal effect on distant cell types in rich medium, could display widely-differing cytotoxicity profiles toward the same cell types in poor medium. This highlights that structurally-related compounds may not only target similar Cdks, but also interact with high affinity with different targets, whose range and concentration would depend on both the cell translational capacity and genotype, that may compete for their interaction with therapeuticallyrelevant Cdk targets.

\section{Materials and methods}

Cell lines and small molecules. The human Saos-2 osteosarcoma and the non-tumorigenic immortalized HaCaT cell line, the untransformed non-immortalized IMR-90 fibroblasts and the carcinoma cell lines MCF-7 (breast), SCC15 (tongue), A431 (vulva epidermoid), SW480 (colon), H1299 (lung), HeLa (cervix) and HBL100 (breast) were grown in Dulbecco's modified Eagle's medium supplied with antibiotics and $10 \%$ foetal calf serum and kept in a $5 \% \mathrm{CO}_{2}$ humidified atmosphere at $37^{\circ} \mathrm{C}$. MCF-7 cells, infected with the E6 oncoprotein of human papillomavirus (HPV) 18, were kindly provided by Reuven Agami (The Netherlands Cancer Institute, Amsterdam, The Netherlands).
Roscovitine, purvalanol A and indirubin-3'-monoxime were from Calbiochem (VWR International S.A.S., France) and the DNA-alkylating agent, mitomycin C, from Sigma (L'Isle d'Abeau Chesnes, France). Preparation of the other 2,6,9-trisubstituted purine derivatives studied has been described elsewhere (15-17).

Cell death analysis. i) Trypan blue assay. In routine experiments, cell viability was estimated by the trypan blue exclusion assay. At the chosen time, the medium containing floating cells was collected and the adhering cells were detached by trypsinization and pooled with the floating ones. An equal volume of a $0.125 \%$ trypan blue solution (Sigma) was then added to an aliquot of the whole suspended cells. Cell viability was assessed by counting under the microscope the proportion of unstained vs. total cells deposited in a hemacytometer. An average value of cell viability was obtained from at least 5 counts over different fields of $\sim 100$ cells; ii) fluorescence and phase contrast observation. Apoptotic cells were detected by labeling with 4',6-diamidino2-phenylindole (DAPI; Sigma) as described before (10). After staining, slides were examined using fluorescence and phase contrast microscopy. The percentage of apoptotic cells, that typically display abnormal chromatin figures and an aberrant morphology (Fig. 2B), was estimated by effecting at least 5 counts over different fields of $\sim 100$ cells. Phase contrast microscopy also permits to see details of the nucleolar organization.

\section{Results}

The cytotoxic effects of roscovitine and their potentiation by mTOR and PI3K inhibitors depend on tumor cell type. 2-(1-ethyl-2-hydroxylamino)-6-benzylamino-9-isopropylpurine (roscovitine) is a potent ATP competitive inhibitor of Cdk2, 5, 7, 9 and Cdk1, 4 (to a lesser extent) (18) that can trigger both G1 and G2/M arrest (9) and apoptosis in various tumor cell types (10,19-24). It also delayed tumor outgrowth in two xenograft models (25). We investigated whether roscovitine in cotreatment with either LY294002 (a direct PI3K inhibitor; 13) or rapamycin (a specific inhibitor of a downtream PI3K target, mTOR; 13) would affect the longterm irreversible cytotoxic effects of roscovitine $(10,11)$. Three human tumor cell lines differing in the $R b / p 53$ status were used: MCF-7 $\left(R b^{+} / p 53^{+}\right)$breast carcinoma cells; MCF7:E6 cells infected with HPV18-E6 oncoprotein (which facilitates wild-type p53 degradation) and Saos-2( $\left.\mathrm{Rb}^{-/} / \mathrm{ps}^{-}\right)$ osteosarcoma cells. Fig. 1 shows that providing MCF-7 cells with $20 \mu \mathrm{M}$ roscovitine under standard growth conditions with $10 \%$ serum and either $100 \mathrm{nM}$ rapamycin or $20 \mu \mathrm{M}$ LY294002 enhanced 3- to 4-fold and 4- to 5-fold, respectively, their death rate at $24 \mathrm{~h}$ as compared to control with $20 \mu \mathrm{M}$ roscovitine alone. In comparison, roscovitine-induced apoptosis, with and without rapamycin, was strongly reduced in MCF-7:E6 and Saos-2 cells. Importantly, MCF-7:E6 and, to a lesser extent, Saos-2 cells remained sensitive to roscovitine/LY294002 cotreatment. Mitomycin C (a DNAalkylating agent that triggers apoptosis via $p 53$-dependent and -independent pathways) killed MCF-7:E6 and MCF-7 cells with equal efficiency. The appearance of its lethal 

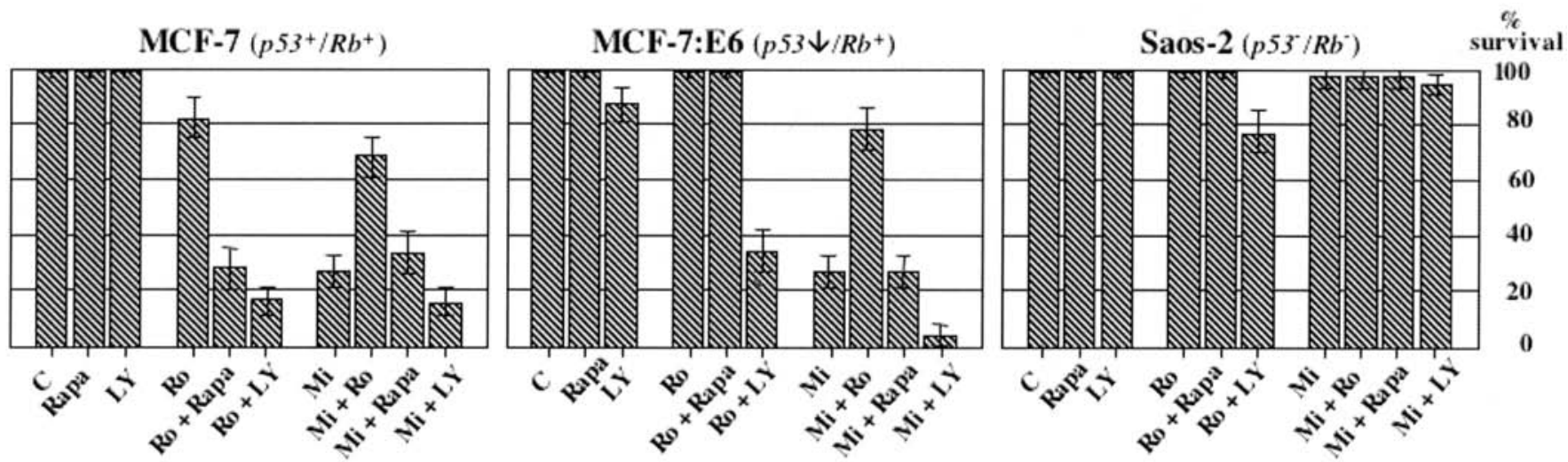

Figure 1. The cytotoxic effects of roscovitine and their potentiation by inhibitors of mTOR and PI3K depend on tumor cell type. The survival rate of MCF-7

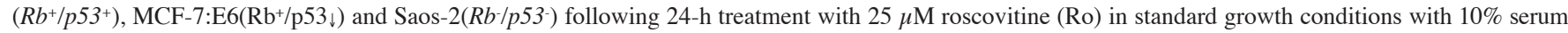
in association with either $20 \mu \mathrm{M} \mathrm{LY} 294002$ (LY) or $100 \mathrm{nM}$ rapamycin (Rapa) was estimated by the trypan blue exclusion assay. The panels show that: i) cotreatment with rapamycin and LY294002 enhances 3- to 4-fold and 4- to 5-fold, respectively, the cytotoxic effects of roscovitine in MCF-7 cells; ii) the lethal effects of roscovitine in MCF-7 cells, with and without rapamycin, depend on p53 as they are strongly reduced in MCF-7:E6 and in Saos-2 osteosarcoma cells; iii) in contrast, LY294002 sensitizes MCF-7:E6 and Saos-2 cells to roscovitine-induced apoptosis; iv) in comparison, the DNA-alkylating compound, mitomycin C (Mi; $7 \mu \mathrm{g} / \mathrm{ml})$, induces the same rate of cell death at $24 \mathrm{~h}$ in MCF-7:E6 and MCF-7 cells; v) however, the appearance of its toxic effects is delayed in the presence of roscovitine and of rapamycin in MCF-7 cells, but not of LY294002; and vi) the Saos-2 cells are much more resistant at early times than MCF-7:E6 cells to p53-independent apoptosis in response to mitomycin C treatment in all conditions, although they eventually die at later times.

effects, however, was delayed upon cotreatment with roscovitine and, albeit to a lesser extent, upon cotreatment with rapamycin in MCF-7 cells. In contrast, LY294002 immediately enhanced the lethal mitomycin $\mathrm{C}$ effects. Saos- 2 cells were much more resistant in the time frame of the experiment than MCF-7:E6 cells to p53-independent apoptosis in response to mitomycin $\mathrm{C}$ treatment in all cases. They still died later on.

Thus, roscovitine is relatively efficient at inducing apoptosis in $\left(R b^{+} / p 53^{+}\right)$tumor cells $(10,11)$ and its cytotoxic effects are enhanced by either mTOR or PI3K inhibitors (which are ineffective on their own). However, it is virtually inactive against $p 53$-deficient, $R b$-proficient or -deficient tumor cells, even in the presence of rapamycin. In contrast, it shows some efficacy against these cells in the presence of LY294002.

Cell susceptibility to the cytotoxic effects of purine derivatives under standard growth conditions depends on both cell type and compound structure. The 2,6,9trisubstituted purines used in this study differ from each other, and from roscovitine, by the presence of either a pyrrolidine methanol or a 3-methylpent-1-yn-3-ol side chain at C-2, and by the nature of the substituents on the C-6 benzylamino/anilino groups (15-17). A systematic screen of a library of 130 compounds was made to compare their relative cytotoxicity toward ten cell lines differing in the $R b / p 53$ status, their tissue origin and their tumorigenic potential. Nineteen compounds were identified as significantly more toxic than roscovitine on the cell types surveyed (Table I). Notable features of the results (Fig. 2A) are: i) the two most sensitive cell lines were $\mathrm{HaCaT}$ and A431 that harbor a p53 mutation (p53*) (HaCaT is a non-tumorigenic keratinocyte line immortalized upon spontaneous acquisition of a p53*; A431 derives from a vulva epidermoid carcinoma); ii) HPV18-infected HeLa cells, in which the degradation rate of p53 and $\mathrm{Rb}$ is enhanced by HPV18-E6/E7 oncoproteins were also very sensitive to all compounds; iii) $\mathrm{MCF}-7\left(\mathrm{Rb}^{+} / \mathrm{p} 53^{+}\right)$and $\mathrm{SCC} 15\left(\mathrm{Rb}^{+} / p 53^{+}\right)$carcinoma cell lines were less responsive than the three above-mentioned, but more so than $\mathrm{SW} 480\left(R b^{+} / p 53^{*}\right)$ and $\mathrm{H} 1299\left(R b^{+} / p 53^{-}\right)$ carcinoma cell lines; iv) the two most resistant tumor cell

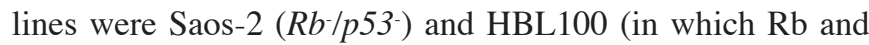
p53 are inactivated by binding to the large T (LT) antigen of simian virus SV40); and v) the 2-pyrrolidine-6-benzylaminopurines 2, 3, 18 and 19 were generally more toxic than the other family members on most cell lines. Compounds of this family were the only ones to induce (like roscovitine; 10) a nuclear p53 accumulation and a nucleolar fragmentation (data not shown).

These observations revealed that roscovitine and members of the new series of purines, could trigger apoptosis in certain tumor cell lines (e.g. HaCaT and A431) by mechanisms other than p53 activation and blockade of ribosome biogenesis. Expectedly, these alternative proapoptotic processes would be repressed in p53-deficient cells in proportion to the extent of corruption of the $\mathrm{Rb}$ pathway and to the consequent up-regulation of rDNA and cell cycle-regulating gene transcription (see Introduction). Saos-2 and HBL100 cell lines were indeed by far the least reactive to all purines.

Furthermore, vi) normal IMR-90 fibroblasts were not completely insensitive to the test compounds, but they were definitely less sensitive than the HaCaT and other tumor cell lines; vii) the 2-alkynyl-6-anilinopurines 15, 16 and 17 displayed abnormally high toxicity toward both Saos-2 and IMR-90 cells; viii) the degree of toxicity of the active compounds did not correlate with their $\mathrm{IC}_{50}$ against starfish oocyte Cdk1-cyclin B, in all cell types examined; and viiii) mitomycin $\mathrm{C}$ did not exhibit such a wide efficacy difference as the purine derivatives toward the various cell types. However, with the exception of the HaCaT and A431, it was definitely more toxic in $p 53$-proficient than in $p 53$ - 
Table I. Structure and $\mathrm{IC}_{50}$ in $\mu \mathrm{M}$ against starfish oocyte cyclin B-Cdk1 of the compounds mentioned in the text.

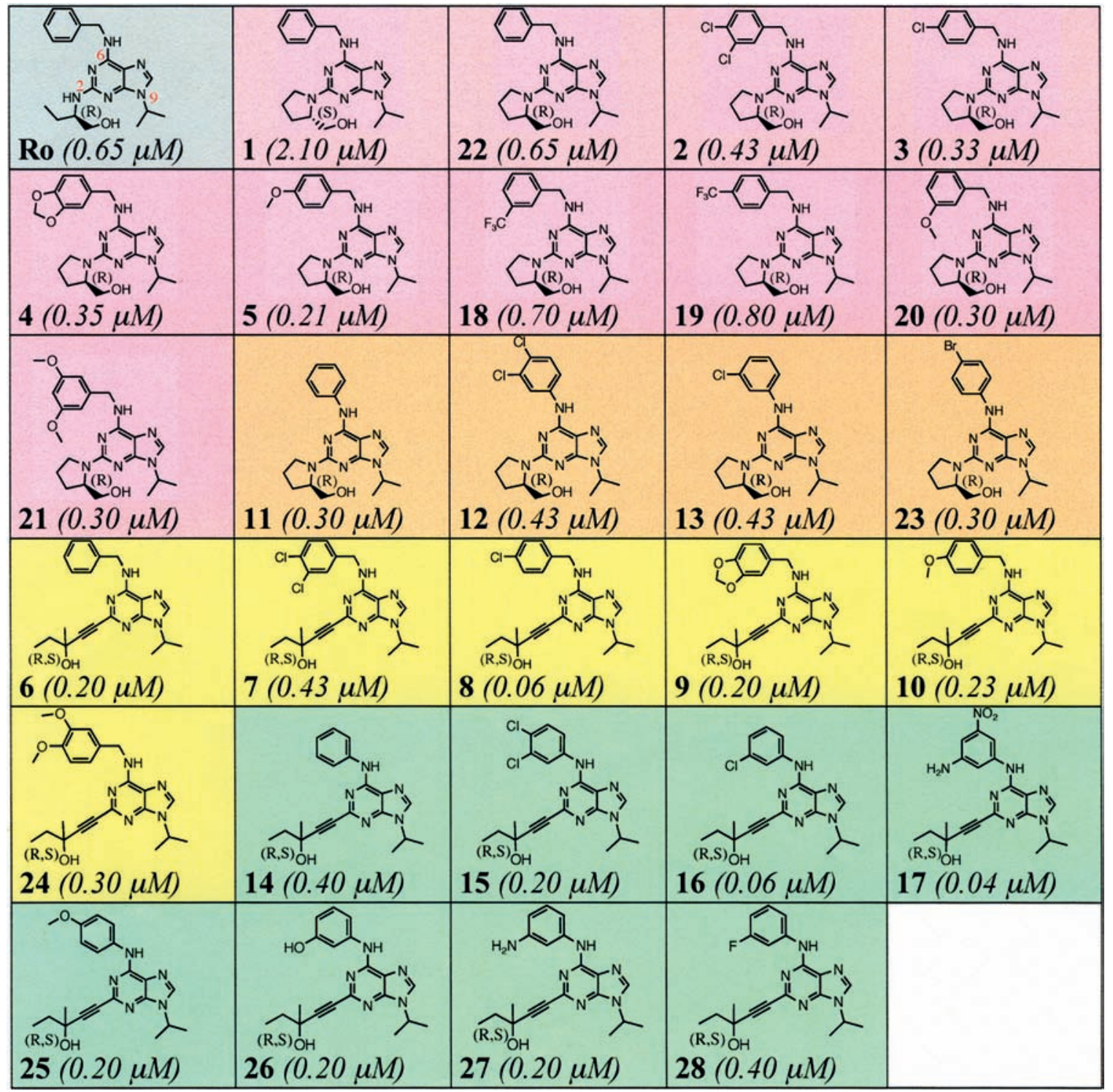

In the grey box is shown the structure of roscovitine (Ro); in pink boxes are shown structures of 6-benzyl-2-pyrrolidine methanol derivatives $(1,2,3,4,5,18,19,20,21$ and 22); in amber boxes, structures of 6-benzyl-2-alkynyl derivatives (11, 12, 13 and 23); in yellow boxes, structures of 6-benzyl-2-alkynyl derivatives $(6,7,8,9,10$ and 24); in green boxes, structures of 6-phenyl-2-alkynyl derivatives (14, 15, 16, $17,25,26,27$ and 28).

deficient cells, and it was slightly less toxic toward normal IMR-90 fibroblasts than toward Saos-2 cells.

Structure-cytotoxicity profiles of C-6 benzylaminopurines under standard (10\% serum) and low serum (0.5\%) conditions (Fig. $3 \mathrm{~A}$ and Table I). The observation that the new series of purines could induce apoptosis via different mechanisms suggested that they could display variable efficacy in combination with treatments that interfere with cell signaling at the level of or upstream from PI3K. This hypothesis was tested by comparing their relative toxicity on MCF-7, Saos-2 and IMR-90 populations provided two days earlier with either 10 or $0.5 \%$ serum. At $40 \mu \mathrm{M}$, the 2-pyrrolidine-6-benzylaminopurines (which were slightly more toxic than roscovitine at $24 \mathrm{~h}$ on $\mathrm{MCF}-7$ populations fed with $10 \%$ serum) were significantly more active on populations fed with $0.5 \%$ serum. Such an improvement in cytotoxicity in low serum was even more marked for some compounds in Saos-2 and IMR-90 populations, although it varied from one cell type to the other depending on the benzylamino substituent. Thus, the aryl halide derivatives 2, 3, 18 and 19, that were the most potent on most cell lines in preliminary assays (Fig. 2A), displayed very high toxicity in low serum on the three cell types and their toxic effects were hardly reduced at $20 \mu \mathrm{M}$ (shown for 2 and 3 ). However, the unsubstituted compound 1 and its R-enantiomer 22, like the 4-OMe (5), 3-OMe (20), and 3,5-dimethoxy (21) derivatives, which were slightly less toxic than the aryl halides on MCF-7 cells in high and low serum at $40 \mu \mathrm{M}$, were definitely less toxic on Saos-2, and especially on IMR- 


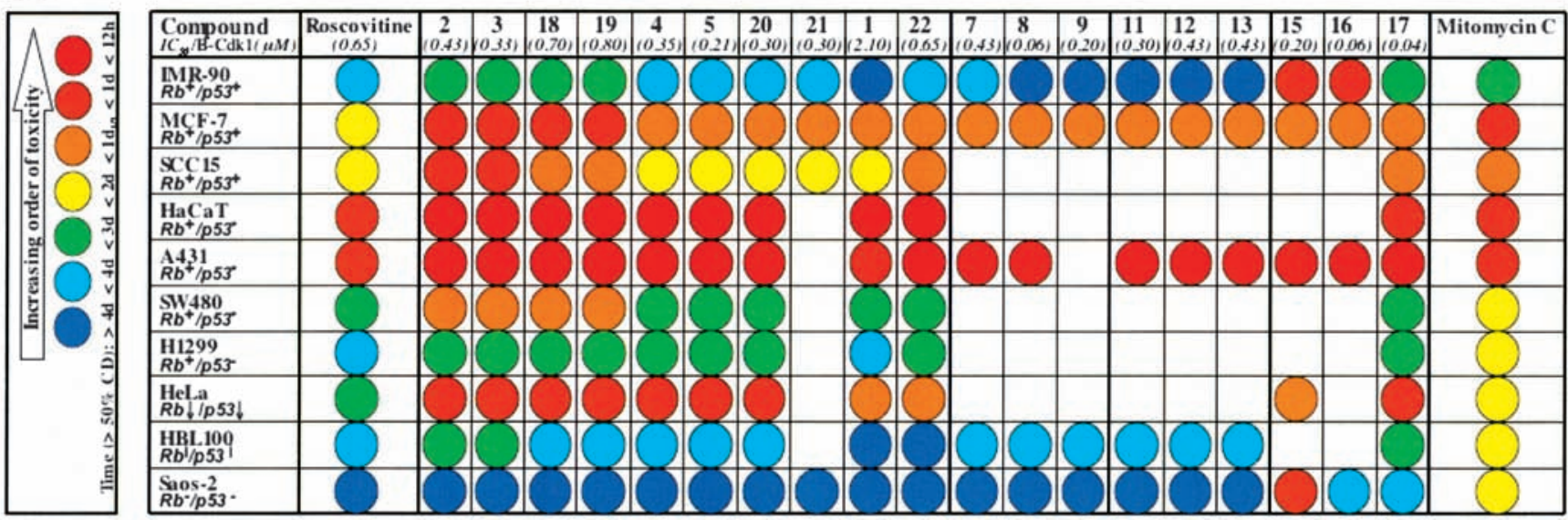

(B)

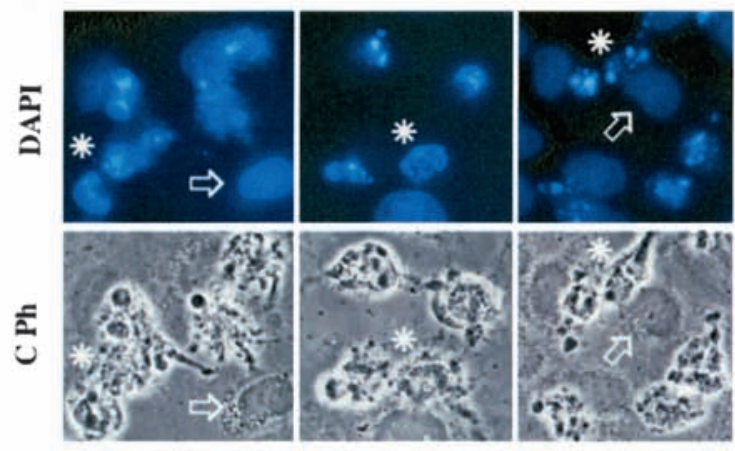

90 cells in low serum (shown for 1 and 5). Noticeably, the cytotoxicity profile for the dioxolane derivative 4 was similar to that for these analogues at $20 \mu \mathrm{M}$, but closer to that for the aryl halides at $40 \mu \mathrm{M}$.

Strikingly, the lethal effects of the 2-alkynyl analogues were systematically weaker in both high and low serum than those of the corresponding 2-pyrrolidine derivatives. Thus, the unsubstituted compound $6\left(\mathrm{IC}_{50}: 0.20 \mu \mathrm{M}\right)$ was essentially inert in high and low serum on the three cell types, although it is a more potent in vitro inhibitor than 1 $\left(\mathrm{IC}_{50}: 2.10 \mu \mathrm{M}\right)$ against starfish oocyte cyclin B-Cdk1. Also, the 3,4-dichloro derivative $\left(7 ; \mathrm{IC}_{50}: 0.43 \mu \mathrm{M}\right)$, that was somewhat less active at $40 \mu \mathrm{M}$ in high serum than 2 on the three cell types, was as effective as 2 in low serum. However, at $20 \mu \mathrm{M}$, a more substantial decline of its efficacy in low serum in tumor-derived than in normal cells was observed. The 3-chloro analogue $\left(8 ; \mathrm{IC}_{50}: 0.06 \mu \mathrm{M}\right)$, which is 7 -fold more potent than 7 against starfish oocyte cyclin B-Cdk1, was markedly less toxic in low serum. As for the corresponding 2-pyrrolidine derivatives, the activity of the aryl oxygen-containing 2-alkynyl analogues decreased in the order $9>10>24$ (3,4-di-OMe; data not shown). Finally, note that roscovitine exhibited cytotoxicity profiles intermediate between those of 1 and 6 .

Structure-cytotoxicity profiles of C-6 anilinopurines and indirubin-3'-monoxime under standard (10\% serum) and low serum $(0.5 \%)$ conditions (Fig. $3 B$ and Table I). The overall cytotoxicity profiles for the three 2-pyrrolidine-6-anilinopurines examined resembled those for the 2-pyrrolidine-
Figure 2. The susceptibility of cells to the cytotoxic effects of purine derivatives depends on both cell type and compound structure under standard growth conditions. (A) A library of 130 2,6,9-trisubstituted purines was screened on ten human cell types differing by the status of the $\mathrm{Rb}$ and p53 proteins (proficient (+), deficient (-), inactivated (i), mutated (*), downregulated $(\downarrow)$, their tissue origin and tumorigenic potential: the untransformed non-immortalized IMR-90 fibroblasts, the carcinoma MCF-7 (breast), SCC15 (tongue), A431 (vulva epidermoid), SW480 (colon), H1299 (lung), HeLa (cervix) and HBL100 (breast) cell lines; the Saos-2 osteosarcoma and the non-tumorigenic immortalized HaCaT cell lines. Nineteen compounds were more toxic on average than roscovitine on most cell types assessed at $25 \mu \mathrm{M}$ concentration. The degree of toxicity of the active compounds is symbolized by a color-coding where a specific color reflects the time of drug exposure required to reach a ratio of dead cells $(\mathrm{CD})>50 \%$ (the code correspondence is indicated in the box). The structure of the purines (and their $\mathrm{IC}_{50}$ against starfish oocyte cyclin B-Cdk1) is shown in Table I. (B) Detection of apoptotic cells by fluorescence and phase contrast microscopy. Apoptotic cells (designated by asterisks), in contrast to normal cells (designated by open arrows), typically display abnormal chromatin figures (detectable by labeling with 4',6-diamidino-2-phenylindole; DAPI) and an aberrant morphology.

6-benzylaminopurines. The improvement in cytotoxicity provided by serum starvation was highest, and of the same order, in the three cell types for the 3-chloro derivative 13 at $20 \mu \mathrm{M}$. At this concentration, the efficacy in low serum of the unsubstituted (11) and 3,4-dichloro (12) analogues toward the three cell types decreased in the order MCF-7 > Saos-2 > IMR-90. Interestingly, the cytotoxicity profiles for purvalanol A (26) were more similar to those for 11 and 12 than for the 3-chloroanilino purine 13.

As for the four aryl halogen-containing 6-benzylaminopurines, the two chloro-substituted 2-alkynyl-6-anilinopurines 15 and 16 were clearly the most toxic of the anilino compounds on the three cell types in high and low serum. Indeed, the 4-OMe $\left(25 ; \mathrm{IC}_{50}: 0.20 \mu \mathrm{M}\right), 3-\mathrm{OH}\left(26 ; \mathrm{IC}_{50}\right.$ : $0.20 \mu \mathrm{M}), 3-\mathrm{NH} 2\left(27 ; \mathrm{IC}_{50}: 0.20 \mu \mathrm{M}\right)$, and 3-F (28; $\mathrm{IC}_{50}$ : $0.40 \mu \mathrm{M}$ ) anilino analogues (data not shown) and the parent compound 14 were essentially inert up to $40 \mu \mathrm{M}$ in rich and poor medium on the three cell types. The 3-amino5-nitro compound 17 , however, displayed a cytotoxicity profile close to that for 16 . As already mentioned, a unique feature common to all active members of this subfamily is that they were more toxic to normal IMR-90 fibroblasts 


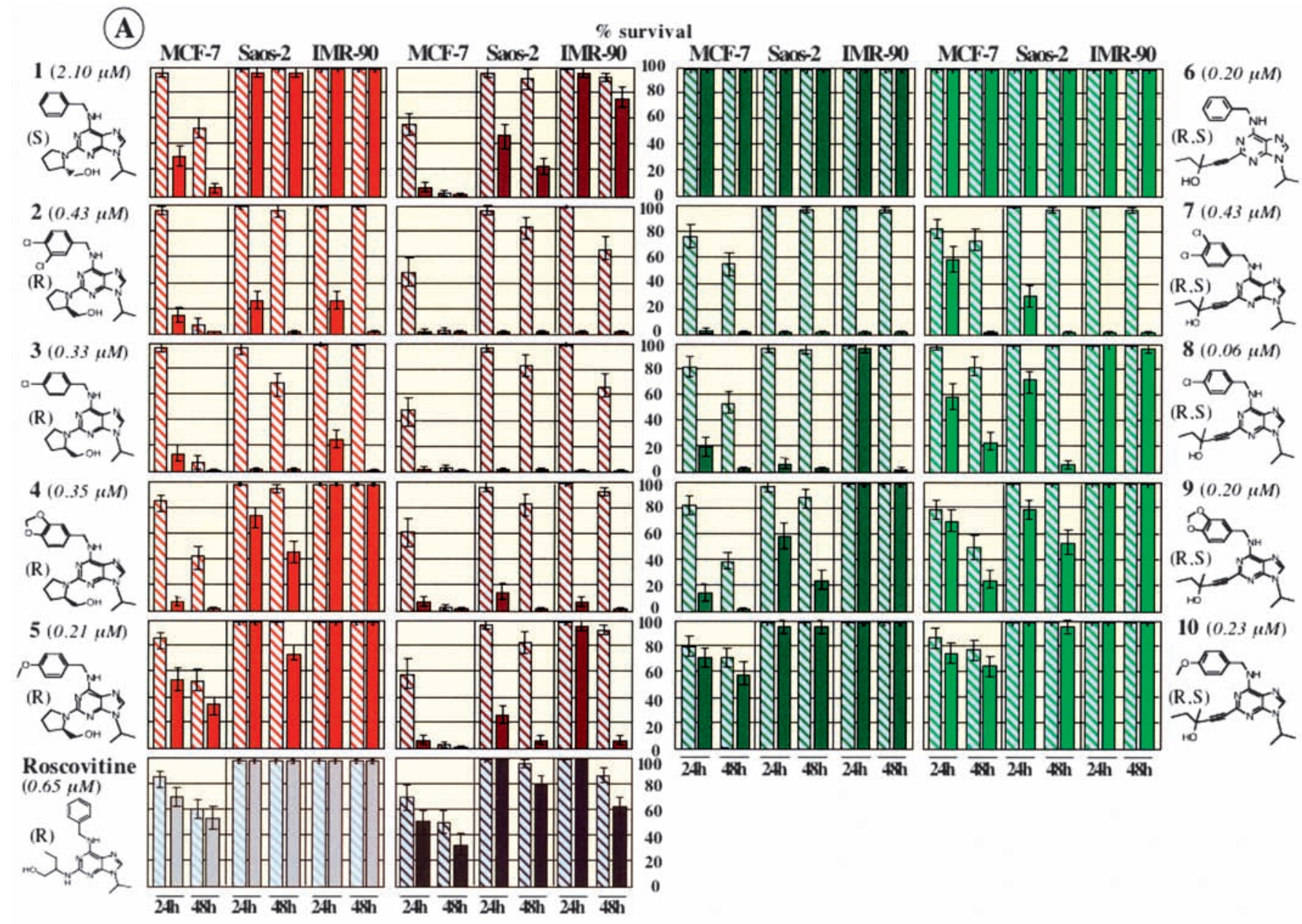

Figure 3. Structure-cytotoxicity profiles of C6 benzylamino/anilinopurines and indirubin-3'-monoxime under standard (10\% serum) and low serum (0.5\%) conditions. A rigorous experimental procedure was followed in order to obtain as meaningful as possible estimates of the relative toxicity of the compounds. Cells collected in a medium containing 5\% serum were plated in 24-well tissue culture plates at two different densities: 6,000 and 8,000 cells per 2-cm diameter well; $24 \mathrm{~h}$ later, the medium was replaced by media containing either $10 \%$ serum in the wells seeded with 6,000 cells or $0.5 \%$ serum in the wells seeded with 8,000 cells; in this way, the cell density at the time of drug application (two days later) was averaging 30,000-35,000 cells in each well. Experiments were performed at two concentrations (20 and $40 \mu \mathrm{M})$. The cell viability was measured by the trypan blue exclusion assay as previously described (10), every day after drug addition over periods of 2 or 3 days for each compound and each cell type selected (the tumor-derived MCF-7 and Saos-2 cell lines and the normal IMR-90 fibroblasts). The results are presented in the form of colored diagrams in which hatched and full columns (whose height reflects the percentage of cell survival) represent high and low serum conditions, respectively. (A) Cytotoxicity profiles after treatment with 2-pyrrolidine-, 2-ethynyl-6-benzylaminopurines and roscovitine are depicted in orange, light green and light grey, respectively, for the $20 \mu \mathrm{M}$ concentration, and in brown, dark green and black, respectively, for the $40 \mu \mathrm{M}$ concentration.

than to tumor-derived MCF-7 and Saos-2 cells in high serum (in which case 15 was also highly toxic on Saos-2 cells).

Remarkably, indirubin-3'-monoxime, which exerts cytostatic effects on many tumor cell types (27), was a much more potent inducer of apoptosis in Saos-2 than in MCF-7 and IMR-90 cells in low serum. Note also that the potentiation by serum privation of mitomycin $\mathrm{C}$-induced apoptosis never attained the extent reached by certain Cdk inhibitors.

\section{Discussion}

The primary aim of this study was to test the prediction that inhibition of the $P I 3 K$ pathway would potentiate the lethal effects of Cdk inhibitors. This prediction is embedded in the idea that corruption of the $R b$ and $p 53$ pathways facilitates tumorigenesis by providing oncogene-bearing cells with a surfeit of Cdk activity and growth, enabling them to elaborate strategies to evade tumor-suppressive pathways and divide in inappropriate settings (28). If so, a potentially universal means to palliate the deficiency of the $R b / p 53$ pathways in cancer cells would be to target both Cdk activities (that help trigger prosurvival pathways or extinguish proapoptotic ones) and their PI3K pathway (that brings the necessary pathway components). The PI3K pathway influences ribosome biogenesis at both the transcriptional and translational level. Its activation leads to cyclin D and Myc stabilization $(29,30)$, and to the subsequent induction of rDNA and $R P$ gene transcription (12,31). Moreover, it boosts the cell translational capacity by promoting the phosphorylation of ribosomal protein S6 kinases and eukaryotic initiation factor 4E-binding proteins (32). Unlike tumor cells, normal cells exposed to a dual treatment with $\mathrm{Cdk}$ and the $P I 3 K$ pathway inhibitors before $\mathrm{R}$ point transit would shift into a resting state whose viability should not rely on Cdks (33), and from which they should readily exit undamaged upon treatment arrest. The above hypothesis also predicts that the growth and survival 
(B)

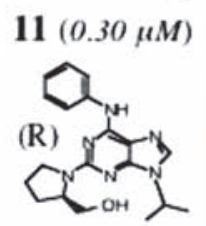

$12(0.43 \mu M)$

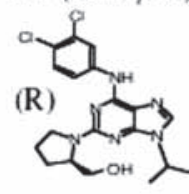

$13(0.43 \mu M)$

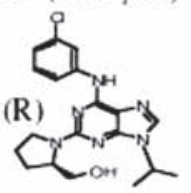

Purvalanol A

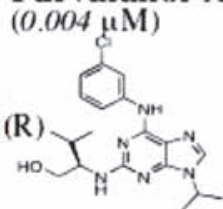

Indirubin

3'-monoxime

$(0.18 \mu M)$

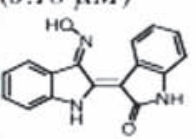

MCF-7 Saos-2 IMR-90
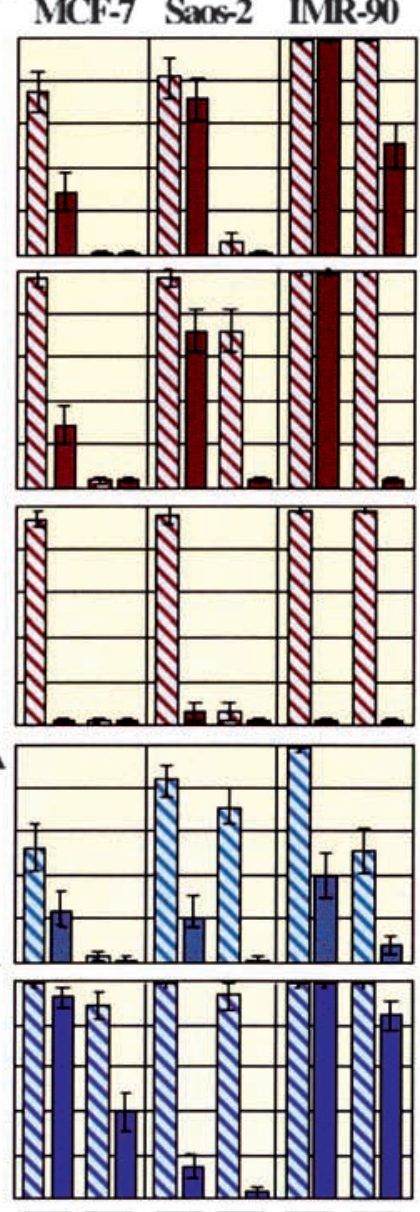

$\overline{24 h} \overline{4 \text { hh }} \overline{24 h} \overline{4 h h} \overline{24 h} \overline{45 h}$

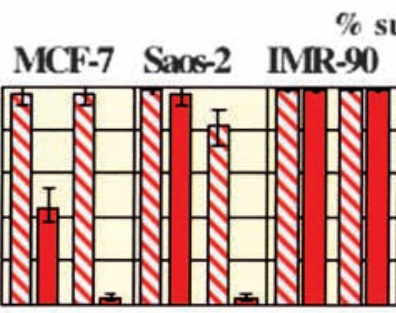

$\%$ survival

MCF-7 Saos-2 IMR-90
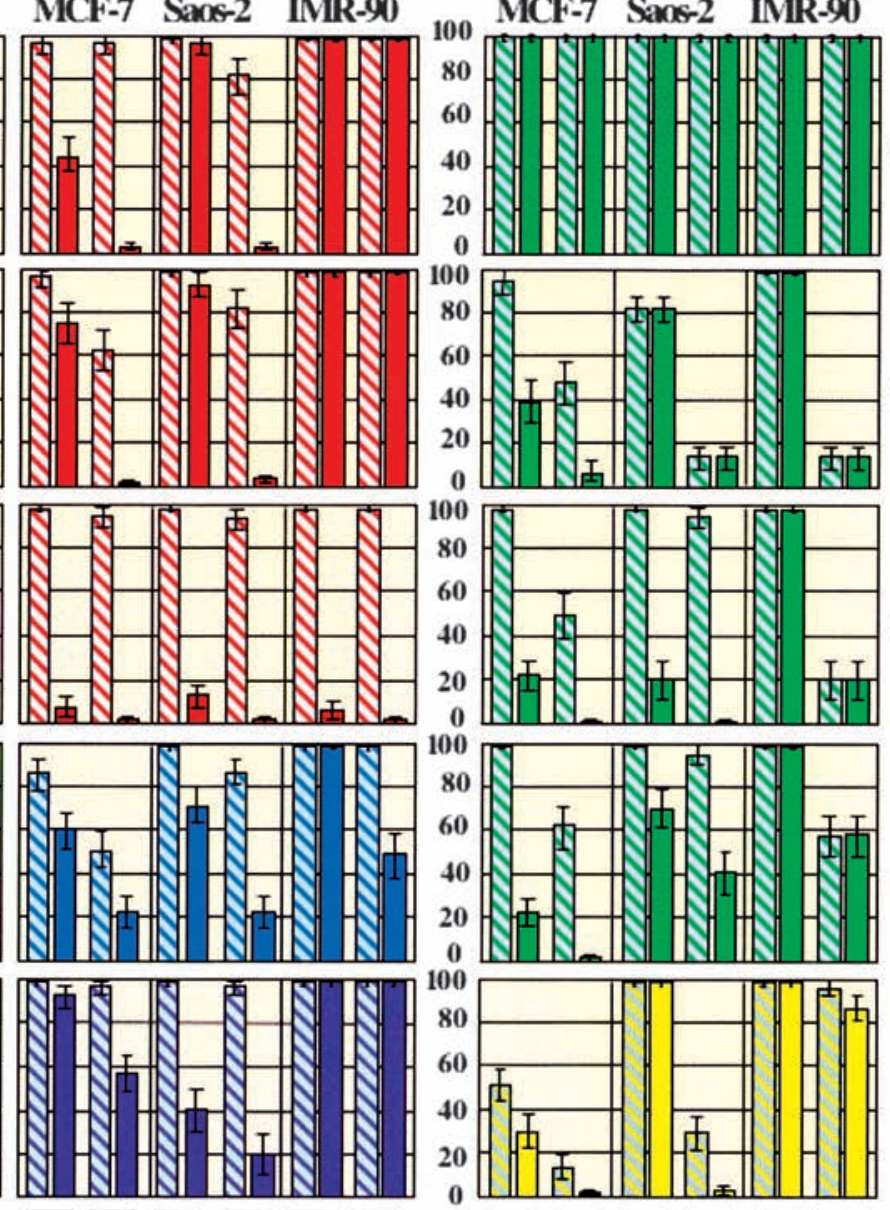

$\overline{24 h} \overline{4 \text { th }} \overline{24 h} \overline{4 h ~} \overline{24 h} \overline{48 h}$ $\overline{24 h} \overline{4 \text { hh }} \overline{24 h} \overline{4 \text { Sh }} \overline{24 h} \overline{4 \$ h}$

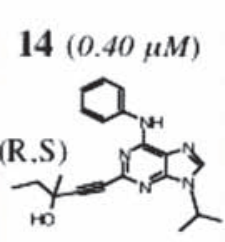

$15(0.20 \mu M)$

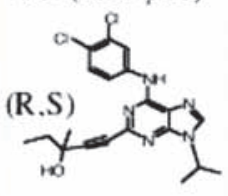

$16(0.06 \mu M)$

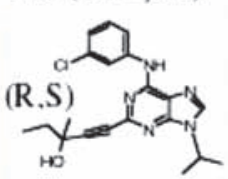

\section{$17(0.04 \mu M)$}

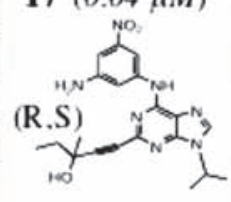

Mitomycin C

(7 $\mu \mathrm{g} / \mathrm{ml})$

Figure 3. Structure-cytotoxicity profiles of C6 benzylamino/anilinopurines and indirubin-3'-monoxime under standard (10\% serum) and low serum (0.5\%) conditions. (B) Cytotoxicity profiles following treatment with $20 \mu \mathrm{M}$ 2-pyrrolidine-, 2-ethynyl-6-anilinopurines, purvalanol A and indirubin-3'-monoxime are depicted in orange, light green, light blue and light violet, respectively, and, after treatment with $40 \mu \mathrm{M} 2$-pyrrolidine-6-anilinopurines, purvalanol A and indirubin-3'-monoxime, in brown, navy-blue and purple, respectively. The yellow diagram presents the cytotoxicity profile produced by $7 \mu \mathrm{g} / \mathrm{ml}$ mitomycin $\mathrm{C}$ in $10 \%$ vs. $0.5 \%$ serum.

capacity of tumor cells would increase with increasing corruption of the $R b / p 53$ pathways.

In fact, Rb-deficient, p53-deficient Saos-2 and HBL100 were the tumor cell lines most resistant to $\mathrm{Cdk}$ inhibitors although they were as sensitive to mitomycin $\mathrm{C}$ as other tumor cell lines whose viability was far more compromised by $\mathrm{Cdk}$ inhibitors. This concurs with the fact that the concentration of mitomycin C primary target (DNA) is independent of the cell growth status. Intriguingly, HPV18-infected HeLa cells were very sensitive to Cdk inhibitors although HPV18-E6/E7 oncoproteins efficiently inactivate p53 and Rb by targeting them to the proteasomes for degradation. Proteasomes, however, are dynamic structures with myriad interacting proteins (34), whose abundance and/or activity could also be regulated by Cdks. In tumors in which the $R b$ pathway is altered via upstream regulators (e.g. cyclin D1 or Cdk4 overexpression or $\mathrm{p} 16^{\text {Ink4a }}$ inactivation), the rate of ribosome synthesis and translation would increase with increasing corruption of cooperative pathways, e.g. the $p 53$ pathway. In fact, $\mathrm{MCF}-7\left(\mathrm{Rb}^{+} / p 53^{+}\right)$and $\mathrm{SCC} 15\left(\mathrm{Rb}^{+} / p 53^{+}\right)$cells were more sensitive to $\mathrm{Cdk}$ inhibitors than $\mathrm{SW} 480\left(R b^{+} / p 53^{*}\right)$ and $\mathrm{H} 1299\left(R b^{+} / p 53^{-}\right)$cells. But, the two cell lines the most sensitive to $\mathrm{Cdk}$ inhibitors were $\operatorname{HaCaT}\left(R b^{+} / p 53^{*}\right)$ and A $431\left(R b^{+} / p 53^{*}\right)$. Expectedly, the chief determinant of $p 53$ deficient cell translational capacity should remain the $R b$ pathway. HaCaT and A431 cells precisely display normal differentiation features in culture, suggestive of a robust $R b$ pathway (35). Thus, the killing efficacy of Cdk inhibitors seems to vary inversely to the extent of up-regulation of ribosome biogenesis and translation imposed by corruption of the $R b / p 53$ pathways, which aligns with our other observation that potentiation of roscovitine-induced apoptosis by the PI3K inhibitor LY294002 decreased in the order MCF-7 > MCF-7:E6 > Saos-2.

These findings concur with reports showing that LY294002 boosts the lethal effects of the pan-Cdk inhibitor flavopiridol (7) in HER2/Neu-overexpressor SKBR3 breast carcinoma cells and in leukemic cells $(23,36)$. Importantly, LY294002 was much more proficient than rapamycin at sensitizing MCF-7:E6 and Saos-2 cells to roscovitine-induced apoptosis, although the two compounds were nearly as active in MCF-7 cells. This argues that the capacity of 
mTOR to help Cdks forward tumor cell survival declines with increasing growth repression by the $R b / p 53$ pathways. This is not unexpected since the relative contribution of mTOR-independent and-dependent mechanisms to the cell translational capacity increases with increasing $P I 3 K$ dependent growth expansion (32).

Cdks, however, participate in physiological processes other than cell cycle regulation (e.g. transcriptional regulation) and their relative implication in each process depends on the cell genotype (18). Therefore, the extent to which deregulated Cdks effectively participate in silencing tumorsuppressive pathways in cancer cells should be determined by the genetic background. Moreover, the cellular effects of a potentially therapeutically-relevant $\mathrm{Cdk}$ inhibitor might be buffered by undesirable off-targets whose range and concentration would also depend on the genetic background (37). The promise of Cdk inhibitor-based anticancer therapies, then, rests upon finding an easy-handling and reliable test to select Cdk inhibitors with the inhibition profile the best suited to eradicate a selected tumor cell type without injuring normal cells. An initially unexpected outcome of this study is that it puts forward such a test.

We found that purines differing by a single substitution, which exerted little lethal effects on distant cell types in rich medium, displayed widely-differing cytotoxicity profiles toward the same cell types in poor medium. For instance, the lethal effects of the aryl halogen-containing 2-pyrrolidine-6-benzylaminopurines 2, 3, 18 and 19 were markedly enhanced in low serum in both normal or tumor cell types. The cytotoxicity profiles for the unsubstituted and aryl oxygen-containing 2-pyrrolidine-6-benzylaminopurines 1 , 4 and 5 were very similar to those for 2, 3, 18 and 19 in high serum and their toxicity against MCF-7 cells in low serum were nearly equal. However, their toxicity was significantly reduced on Saos-2 and even more so on IMR-90 cells in low serum compared to that for the aryl halides. Importantly, a cotreatment with $20 \mu \mathrm{M} \mathrm{LY} 294002$ in high serum gave cytotoxicity profiles similar to those obtained with the compounds alone in low serum (data not shown). In contrast, cotreatment with $100 \mathrm{nM}$ rapamycin did not affect the cytotoxicity profile in high serum of any purine in Saos-2 and IMR-90 cells (data not shown). Serum privation most likely acts upstream of PI3K to lower the concentration of cell constituents, notably of Cdk inhibitor targets. Thereby, it must suppress certain interactions between the inhibitors and low-affinity targets (Cdk or Cdk-unrelated) whose excessive concentration in rich media may compensate for their low affinity, allowing them to compete for the interaction between the inhibitors and their therapeutically-relevant $\mathrm{Cdk}$ targets. The range and concentration of such undesirable targets should increase with increasing corruption of the Rb/p53 pathways. These buffering effects would further raise the extent of repression of the $P I 3 K$ pathway required for the $\mathrm{Cdk}$ inhibitors to reactivate the latent tumor suppressor programmes silenced in cancer cells. Strikingly, sensitization by low serum (or LY294002) to the oxindole indirubin-3'-monoxime was more substantial on Saos-2 than on MCF-7 and IMR-90. Indirubin3'-monoxime, however, is much more efficient at inhibiting glycogen synthase kinase 3 (GSK-3) than cyclin-Cdk1,2 (38). By preventing GSK-3-mediated degradation of cyclin
D1, it should favor the formation of active cyclin DCdk4,6 complexes, enabling them to block the proapoptotic activity of $\mathrm{Rb}$ in $R b$-proficient cells (39). This may be why

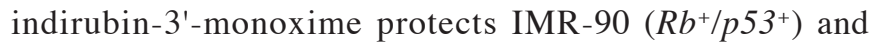
MCF-7 $\left(R b^{+} / p 53^{+}\right)$but not Saos-2(Rb/p53) cells from the toxicity of $\mathrm{Cdk}$ inhibition in low serum.

Thus, in the perspective of clinical development in association with the PI3K pathway inhibitors, it might be advisable to select tumor cell type-specific Cdk inhibitors on the basis of their toxicity in cell-culture-based assays performed at limiting serum concentration, sufficient to suppress their interaction with undesirable off-targets. Potentially valuable molecules should meet the requirement that their lethal effects under restricted growth conditions toward representative normal cell types should be negligible at the minimal dose at which they would already be substantial against one (or several) tumor cell type. Accordingly, compounds 1, 22, 9 and 11 that present the greatest cytotoxicity differential between MCF-7 cells and normal IMR-90 fibroblasts in low serum appear as the most promising of the presently-evaluated molecules against MCF-7-like cancer cells. But, indirubin-3'-monoxime appears as the most valuable compound against Saos-2-like cancer cells. In our tests, these molecules are superior to roscovitine, which is currently undergoing clinical trials.

\section{Acknowledgements}

The study was supported by the Centre National pour la Recherche Scientifique, the Curie Institute and the Association pour la Recherche sur le Cancer (contract \# 4660 to Michel Legraverend).

\section{References}

1. Lowe SW, Cepero E and Evan G: Intrinsic tumor suppression. Nature 432: 307-315, 2004.

2. Sherr CJ and McCormick F: The Rb and p53 pathways in cancer. Cancer Cell 2: 103-112, 2002.

3. Harbour JW, Luo RX, Dei Santi A, Postigo AA and Dean DC: Cdk phosphorylation triggers sequential intramolecular interactions that progressively block $\mathrm{Rb}$ functions as cells move through G1. Cell 98: 859-869, 1999.

4. Vousden $\mathrm{KH}$ and $\mathrm{Lu} \mathrm{X}$ : Live or let die: the cell's response to p53. Nat Rev Cancer 2: 594-604, 2002.

5. Hardcastle IR, Golding BT and Griffin RJ: Designing inhibitors of cyclin-dependent kinases. Annu Rev Pharmacol Toxicol 42: 325-348, 2002.

6. Shapiro GI: Cyclin-dependent kinase pathways as targets for cancer treatment. J Clin Oncol 24: 1770-1783, 2006.

7. Day Y and Grant S: Small molecule inhibitors targeting cyclindependent kinases as anticancer agents. Curr Oncol Rep 6: 123-130, 2004.

8. Phillips AC and Vousden K: E2F-1 dependent apoptosis. Apoptosis 6: 173-182, 2001.

9. Meijer L, Borgne A, Mulner O, Chong JPJ, Blow JJ, Inagaki N, Inagaki M, Delcros J-G and Moulinoux J-P: Biochemical and cellular effects of roscovitine, a potent and selective inhibitor of the cyclin-dependent kinases cdc2, cdk2 and cdk5. Eur J Biochem 243: 527-536, 1997.

10. David-Pfeuty T: Potent inhibitors of cyclin-dependent kinase 2 induce nuclear accumulation of wild-type p53 and nucleolar fragmentation in human untransformed and tumor-derived cells. Oncogene 18: 7409-7422, 1999.

11. David-Pfeuty T, Nouvian-Dooghe Y, Sirri V, Roussel P and Hernandez-Verdun D: Common and reversible regulation of wild-type p53 function and ribosomal biogenesis by protein kinases in human cells. Oncogene 20: 5951-5963, 2001. 
12. Ruggero D and Pandolfi PP: Does the ribosome translate cancer? Nat Rev Cancer 3: 179-192, 2003.

13. Vivanco I and Sawyers CL: The phosphatidylinositol 3-kinaseAkt pathway in human cancer. Nat Rev Cancer 2: 489-501, 2002.

14. Kerbel $\mathrm{R}$ and Folkman $\mathrm{J}$ : Clinical translation of angiogenesis inhibitors. Nat Rev Cancer 2: 93-105, 2002

15. Legraverend M, Ludwig O, Leclerc S and Meijer L: Synthesis of a new series of purine derivatives and their anti-cyclindependent kinase activities. J Heterocyclic Chem 38: 299-303, 2001.

16. Legraverend $M$, Tunnah $\mathrm{P}$, Noble $\mathrm{M}$, Ducrot $\mathrm{P}$, Ludwig O, Grierson DS, Leost M, Meijer L and Endicott J: Cyclindependent kinase inhibition by new C-2 alkynylated purine derivatives and molecular structure of a CDK2-inhibitor complex. J Med Chem 43: 1282-1292, 2000.

17. Ducrot $P$, Legraverend $M$ and Grierson DS: D-QSAR CoMFA on cyclin-dependent kinase inhibitors. J Med Chem 43: 4098-4108, 2003.

18. Fischer PM: The use of Cdk inhibitors in oncology: a pharmacological perspective. Cell Cycle 3: 742-746, 2004.

19. Mgbonyebi OP, Russo J and Russo IH: Roscovitine induces cell death and morphological changes indicative of apoptosis in MDA-MB-231 breast cancer cells. Cancer Res 15: 1903-1910, 1999.

20. Edamatsu H, Gau C-L, Nemoto T, Guo L and Tamanoi F Cdk inhibitors, roscovitine and olomoucine, synergize with farnesyltransferase inhibitor (FTI) to induce efficient apoptosis of human cancer cells. Oncogene 19: 3059-3068, 2000.

21. Somerville L and Cory JG: Enhanced roscovitine-induced apoptosis is mediated by a caspase-like activity in deoxyadenosine-resistant mouse leukemia L1210 cells. Anticancer Res 20: 3347-3355, 2000.

22. Mihara M, Shintani S, Kiyota A, Matsumura T and Wong DT: Cyclin-dependent kinase inhibitor (roscovitine) suppresses growth and induces apoptosis by regulating $\mathrm{Bcl}-\mathrm{x}$ in head and neck squamous carcinoma cells. Int J Oncol 21: 95-101, 2002.

23. Yu C, Rahmani M, Dai Y, Conrad D, Krystal G, Dent P and Grant S: The lethal effects of pharmacological cyclin-dependent kinase inhibitors in human leukemia cells proceed through a phosphatidylinositol 3-kinase/Akt-dependent process. Cancer Res 63: 1822-1833, 2003.

24. Hahntow IN, Schneller F, Oelsner M, Weick K, Ringhausen I, Fend F, Pescel, C and Decker T: Cyclin-dependent kinase inhibitor Roscovitine induces apoptosis in chronic lymphocytic leukemia cells. Leukemia 18: 747-755, 2004.

25. McClue SJ, Blake D, Clarke R, Cowan A, Cummings L, Fisher PM, MacKenzie M, Melville J, Stewart K, Wang S, Zhelev N, Zheleva D and Lane D: In vitro and in vivo antitumor properties of the cyclin dependent kinase inhibitor CYC202 (roscovitine). Int J Cancer 102: 463-468, 2002.
26. Gray NS, Wodicka L, Thunnissen A-MWH, Norman, TC, Kwon S, Espinoza FH, Morgan DO, Barnes G, LeClerc S, Meijer L, Kim S-H, Lockhart DJ and Schultz PG: Exploiting chemical libraries, structure, and genomics in the search for kinase inhibitors. Science 281: 533-538, 1998.

27. Hoessel R, Leclerc S, Endicott JA, Nobel MEN, Lawrie A, Tunnah P, Leost M, Damiens E, Marie D, Marko D, Niederberger E, Tang W, Eisenbrand G and Meijer L: Indirubin, the active constituent of a chinese antileukaemia medicine, inhibits cyclin-dependent kinases. Nat Cell Biol 1: 60-67, 1999.

28. David-Pfeuty T: The flexible evolutionary anchorage-dependent Pardee's restriction point of mammalian cells. How its deregulation may lead to cancer. BBA-Rev Cancer 1765: 38-66, 2006.

29. Diehl JA, Cheng M, Roussel MF and Sherr CJ: Glycogen synthase kinase- 3 B regulates cyclin D1 proteolysis and subcellular localization. Genes Dev 12: 3499-3511, 1998.

30. Sears R, Nuckolls F, Haura E, Taya Y, Tamai K and Nevins JR: Multiple Ras-dependent phosphorylation pathways regulate Myc protein stability. Genes Dev 14: 2501-2514, 2000.

31. Boxer LM and Dang CV: Translocations involving c-myc and c-myc functions. Oncogene 20: 5595-5610, 2001.

32. Fingar DC and Blenis J: Target of rapamycin (TOR): an integrator of nutrient and growth factor signals and coordinator of cell growth and cell cycle progression. Oncogene 23: 3151-3171, 2004

33. Pardee AB and James LJ: Selective killing of transformed baby hamster kidney (BHK) cells. Proc Natl Acad Sci USA 72: 4994-4998, 1975.

34. Glickman MH and Raveh D: Proteasome plasticity. FEBS Lett 579: 3214-3223, 2005.

35. Lipinski MM and Jacks T: The retinoblastoma gene family in differentiation and development. Oncogene 18: 7873-7882, 1999.

36. Wu K, Wang C, D'Amico M, Lee RJ, Albanese C, Pestell RG and Mani S: Flavopiridol and trastuzumab synergistically inhibit proliferation of breast cancer cells: association with selective cooperative inhibition of cyclin D1-dependent kinase and Akt signaling pathways. Mol Cancer Ther 1: 695-706, 2002.

37. Knight ZA and Shokat KM: Features of selective kinase inhibitors. Chem Biol 12: 621-637, 2005.

38. Polychronopoulos P, Magiatis P, Skaltsounis A-L, Myrianthopoulos V, Mikros E, Tarricone A, Musacchio A, Roe SM, Pearl L, Leost M, Greengard P and Meijer L: Structural basis for the synthesis of indirubins as potent and selective inhibitors of glycogen synthase kinase-3 and cyclin-dependent kinases. J Med Chem 47: 935-946, 2004.

39 Liu H, Dibling B, Spike B, Dirlam A and Macleod K: New roles for the $\mathrm{Rb}$ tumor suppressor protein. Curr Opin Genet Dev 14: 55-64, 2004. 